\title{
Publications électroniques et revues savantes : acteurs, rôles et réseaux
}

\section{Electronic Publications and Scholarly Journals: Roles and Networks \\ Publicaciones electrónicas y revistas académicas: actores, roles y redes}

\section{Gérard Boismenu et Guylaine Beaudry}

Volume 45, numéro 4, octobre-décembre 1999

Édition électronique

URI : https://id.erudit.org/iderudit/1032718ar

DOI : https://doi.org/10.7202/1032718ar

Aller au sommaire du numéro

\section{Éditeur(s)}

Association pour l'avancement des sciences et des techniques de la documentation (ASTED)

\section{ISSN}

0315-2340 (imprimé)

2291-8949 (numérique)

Découvrir la revue

Citer cet article

Boismenu, G. \& Beaudry, G. (1999). Publications électroniques et revues savantes : acteurs, rôles et réseaux. Documentation et bibliothèques, 45(4), 149-159. https://doi.org/10.7202/1032718ar
Résumé de l'article

Dans l'ensemble des possibilités offertes aux chercheurs pour la communication des résultats de recherche, la revue savante (électronique ou imprimée) est un maillon important du système de communication scientifique. À partir d'un échantillon de 200 revues dominantes dans huit disciplines, il est démontré que les prix exorbitants pratiqués par les éditeurs commerciaux ne sont pas en relation avec les revues ayant des facteurs d'impact élevés. Les « lieux d'édition sans but lucratif », que ce soit les associations, les presses universitaires ou les bibliothèques, occupent un espace stratégique. Il importe d'établir des stratégies d'action visant la mise en réseau de ces « lieux de publication ».
Tous droits réservés $@$ Association pour l'avancement des sciences et des techniques de la documentation (ASTED), 1999
Ce document est protégé par la loi sur le droit d'auteur. L'utilisation des services d'Érudit (y compris la reproduction) est assujettie à sa politique d'utilisation que vous pouvez consulter en ligne.

https://apropos.erudit.org/fr/usagers/politique-dutilisation/ 


\title{
Publications électroniques et revues savantes: acteurs, rôles et réseaux*
}

\author{
Gérard Boismenu \\ Professeur titulaire en science politique et \\ Directeur scientifique des Presses de l'Université de Montréal \\ gerard.boismenu@montreal.ca
}

Guylaine Beaudry

Chef de service - édition électronique

Service des bibliothèques de l'Université de Montréal

Presses de l'Université de Montréal

guylaine.beaudry@umontreal.ca

Dans l'ensemble des possibilités offertes aux chercheurs pour la communication des résultats de recherche, la revue savante (électronique ou imprimée) est un maillon important du système de communication scientifique. À partir d'un échantillon de 200 revues dominantes dans huit disciplines, il est démontré que les prix exorbitants pratiqués par les éditeurs commerciaux ne sont pas en relation avec les revues ayant des facteurs d'impact élevés. Les «lieux d'édition sans but lucratif ", que ce soit les associations, les presses universitaires ou les bibliothèques, occupent un espace stratégique. Il importe d'établir des stratégies d'action visant la mise en réseau de ces « lieux de publication".

\begin{abstract}
Electronic Publications and Scholarly Journals: Roles and Networks

Among the various options available to researchers who wish to communicate the results of their findings, the scholarly journal (electronic or paper format) is an important part of scientific communication. Using a sample of 200 major journals in eight fields, it was found that the exorbitant prices demanded by commercial publishers do not correspond to journals with high impact. The «notfor-profit " publishers, be they associations, university presses or libraries, play significant roles. It therefore becomes necessary to establish strategic actions that allow this type of publisher to create networks.
\end{abstract}

Publicaciones electrónicas y revistas académicas: actores, roles y redes

Dentro del conjunto de posibilidades ofrecidas a los investigadores para comunicar los resultados de sus investigaciones, la revista académica (electrónica o impresa) constituye un eslabón importante del sistema de comunicaciones científicas. A partir de una muestra de 200 revistas importantes que tocan ocho disciplinas, se ha demostrado que los precios exorbitantes aplicados por los editores comerciales no están en relación con las revistas cuyos factores de repercusión son elevados. Las «casas de edición sin fines de lucro", se trate de asociaciones, editoriales universitarias o bibliotecas, ocupan un lugar clave. Es importante establecer estrategias de acción destinadas a formar una red con estas «casas de edición»
Le système de communication scientifique et, en particulier, le rôle que les revues savantes y jouent, traversent une période de bouleversement. De ce constat, plusieurs souligneront le caractère critique de la situation. On pourrait mettre en cause la revue comme vecteur de communication des résultats de recherche, mais le bilan à dresser sur ce plan exigerait beaucoup de nuances et il serait présomp- tueux de conclure par une condamnation sans appel (Tenopir et King 1998).

Le problème se présente sous sa forme exacerbée par la spirale inflationniste des prix des abonnements des revues qui met dans une situation intenable les institutions universitaires et de recherche. Avec des ressources qui stagnent, elles doivent faire face à une vertigineuse croissance des prix, bien au-delà de l'inflation et de l'évolution des paramètres qui caractérisent les revues. La compréhension de ce cercle vicieux est bien documentée et on peut en observer les effets.

Autant la notion de revue est une notion vague et abstraite, dans la mesure où, sous ce vocable, elle renvoie à des réalités extrêmement différentes, autant, pour tenir compte des conditions de commercialisation, il faut qualifier les structures socia-

* Nous exprimons nos plus sincères remerciements à Brigitte Gemme, associée au Centre interuniversitaire de recherche sur la science et la technologie (CIRST.), qui a contribué très efficacement à rassembler la documentation et les données pour la présente étude. Nous tenons de plus à remercier Albert Tabah de l'EBSI concernant une première version de cet article. Bien entendu, nous restons les seuls responsables de ce texte. 
les et économiques dans lesquelles s'inscrivent les revues savantes. Ce n'est que de cette façon que l'on peut aborder certaines manifestations comme la variation dans les augmentations pratiquées selon les types d'éditeurs, le contraste frappant dans le taux de croissance des prix selon les secteurs de la connaissance, ou le fait que les tarifs d'abonnement les plus élevés connaissent une croissance des prix nettement plus forte.

Le monde des revues savantes est fortement structuré, et cela au moins de deux points de vue. D'abord, les réseaux de communication scientifique sont institutionnalisés et épousent une hiérarchie seIon laquelle, en cascade, un ordre de préséance et de statuts est établi entre les revues savantes, ce qui leur confère, à des degrés différents, une validité, une légitimité, un rayonnement et un impact. Cette structuration des réseaux de communication n'est pas homogène selon les secteurs; on peut suggérer, par exemple, que dans les sciences humaines et sociales où les paradigmes, souvent en compétition, ne sont pas solidement établis, cette structuration est plus poreuse alors qu'à l'inverse, dans les secteurs des sciences, des techniques et de la médecine, elle est beaucoup plus dense et impérative. Cela permet sans doute de comprendre pourquoi, dans ces derniers secteurs plus particulièrement, les grandes revues qui font autorité (qualité de la recherche, reconnaissance institutionnelle, rayonnement) s'imposent en dépit d'autres considérations.

Ensuite, l'univers structuré et structurant, c'est celui de l'organisation économique et corporative qui supporte l'édition universitaire. De grands oligopoles dominent largement la production et la diffusion des revues, particulièrement dans les secteurs où la hiérarchisation des réseaux de la communication scientifique est la plus poussée. Les éditeurs commerciaux contrôlent $40 \%$ des 6771 revues scientifiques aux États-Unis, mais jouissent d'un poids nettement plus considérable, si on tient compte des ventes; les sociétés savantes produisent le quart des revies, alors que les presses universitaires et les organismes de recherche (publics ou privés) les $35 \%$ qui restent (Abate 1997).

Or, les prix pratiqués par les grands oligopoles leur permettent de faire des affaires très lucratives, au point que les publications universitaires constituent l'un des segments les plus profitables de l'ensemble de leurs opérations. La mesure des prix administrés peut être révélée par l'étude de Wyly qui prend l'année 1997 comme référence. II établit que si Reed Elsevier, Wolters Kluwer et Plenum Publishing s'étaient limités à pratiquer une marge nette de profit sur les ventes comparable à la médiane dans la branche de la publication des périodiques, dans le seul domaine des publications universitaires, les clients auraient épargné quelque 240 millions de dollars américains (Wyly 1998).

Les deux niveaux de structuration ont des effets cumulatifs permettant à certaines grandes compagnies de toucher une rente de situation particulièrement importante, ce qui exacerbe la crise financière des bibliothèques et fragilise les modes actuels de communication scientifique. Par ailleurs, si, dans le contexte économique évoqué, certaines sociétés savantes éditrices sont aussi en mesure de toucher une rente de situation pour leurs propres revues et d'en tirer des bénéfices utilisés à leur vie organisationnelle, on comprendra que généralement les organismes sans but lucratif qui œuvrent dans le domaine de l'édition universitaire n'ont pas accès à cette assiette au beurre.

Les gisements de richesse et les marges bénéficiaires confortables sont bien étrangers à la plupart des acteurs autres que les grands oligopoles de l'édition universitaire et ne sauraient caractériser le secteur en général. Pour y voir plus clair, nous allons d'abord situer la revue savante dans le système de communication scientifique afin d'en souligner la mission spécifique. Partant de là, nous allons insister sur la nécessité de cibler les attaques contre les prix administrés et de mettre en situation la discussion sur les structures de coût de l'édition savante. La cherté des abonnements tient, pour une part, à une structure de coûts élevés et, pour une part appréciable, aux marges que peuvent toucher les oligopoles. II faut maintenant isoler la première dimension, pour s'interroger sur les usages de la communication scientifique selon les secteurs en considérant particulièrement les dimensions socioéconomiques. Ces considérations nous amèneront à dépasser un niveau macroscopique et à proposer une stratégie d'action basée sur les structures existantes.

\section{Les revues et les modèles de communications scientifiques}

\section{Structuration sociologique des disciplines}

Les travaux de Becher (1987) ont permis d'identifier plusieurs caractéristiques propres au fonctionnement des chercheurs des disciplines en sciences et en sciences humaines et sociales. Ces distinctions sont fondamentales et expliquent les modèles développés par le passé ainsi que les nouveaux systèmes de communication émergents. Sans compter les distinctions d'ordre méthodologique, de mobilité ou d'initiation des jeunes chercheurs, l'interaction sociale et les modes de communication des deux types de communauté justifient l'existence de systèmes distincts de diffusion des résultats de la recherche.

Les collèges invisibles en sciences ont une importance cruciale dans l'échange continuel d'information, de prépublications et de rapports de recherche. Le haut ratio «problème-chercheurs " crée une compétition très grande. Cette situation fait en sorte que la demande (et l'offre) de rapports fréquents sur les progrès est forte, nécessitant des voies rapides (et larges) de communication, que ce soit par la voie des conférences (plus nombreuses et plus courues qu'en sciences sociales) ou des revues. Ces dernières ont donc des durées courtes d'arbitrage des articles, variant sous la barre des 6 mois, et on retrouve quelques hebdomadaires au nombre des revues scientifiques qui sont souvent parmi les plus citées, dont Science et Nature. Toutefois, l'échange de prépublications est le mode de communication privilégié et les visites entre collègues sont très fréquentes permettant d'obtenir les réactions immédiates et l'échange de détails qu'on ne publie pas.

La langue des sciences est également un facteur différenciateur. Hacking (1996) fait la démonstration de l'inaccessibilité de certains textes scientifiques en citant un extrait d'un article en neurophysique: «If postsynaptic adrenergic neurons in neonatal rats were chemically destroyed with 6-hydroxyudopamine [...] the 
normal development of presynaptic ChAc activity was prevented." (Hacking 1996). La compréhension de cette phrase par un lecteur non initié se limite à l'analyse grammaticale.

Dès qu'on quitte le noyau dur des sciences et au fur et à mesure qu'on se déplace vers les sciences appliquées et les sciences sociales, la pression des courtes périodes avant publication diminue (Becher 1987, 285). La fréquentation des conférences internationales est moins grande et sert davantage à témoigner une solidarité sociale entre chercheurs d'une même discipline. Le chercheur est plutôt au courant des développements de sa discipline par la lecture des publications des collègues. II n'est pas rare que la publication d'un article de revue prenne une année et que celle des livres peut prendre jusqu'à deux ans.

\section{Différents modèles de communication scientifique}

Bien avant Internet, la communication scientifique se faisait selon plusieurs modèles. $A$ fortiori, les nouvelles technologies de l'information ont multiplié les modèles d'échange d'information. Les chercheurs des disciplines scientifiques confirment l'importance des collèges invisibles et des communications personnelles par l'utilisation du courriel et des serveurs de prépublications par lesquels les collèges invisibles se voient démocratisés, ce qui change radicalement les règles de diffusion de la recherche. Cependant, ce modèle proposé par Ginsparg ${ }^{1}$ et implanté avec un succès retentissant dans la communauté des chercheurs en physique des hautes énergies, tout en faisant fi du rattachement des revues à une école ou à un courant particulier, se fait reprocher par plusieurs son effet centralisateur (Blumenstyk et Kiernan 1999). Le contrôle de qualité ainsi que l'archivage des documents déposés sur ces serveurs posent également problème (Rowland 1997) puisque les fichiers sont conservés tels que les auteurs les ont transmis, sans évaluation formelle par les pairs et sans conversion des textes et des données vers un format normalisé.

Toutefois, comme le note Le Crosnier: «[...] dans certaines communautés, quand l'urgence de la publication prime, quand le réseau scientifique est peu étendu et bien structuré, les prépublications gardent une place centrale pour la reconnaissance. » (Le Crosnier 1997). Retraçant l'historique de la communication scientifique, de la lettre au livre imprimé, du livre à l'article de revue et de la revue aux bases de données bibliographiques, De Solla Price conclut en notant: "At turn [...] all the old mechanisms were preserved but new technical advances have modified the whole system and produced new reactions, new forces» (Price 1967, 201). D'autres serveurs de prépublications sont mis en place, notamment celui proposé par Stevan Harnad en sciences cognitives ${ }^{2}$ et, récemment, le National Institute of Health des États-Unis annonçait la création d'un tel serveur en recherche biomédicale. Reste à voir si ce modèle, que certains qualifient de «subversif» (Harnad 1997), peut s'appliquer à tous les domaines et répondre aux besoins du milieu universitaire.

Les communications facilitées par le courriel et les serveurs de prépublications ainsi que les possibilités des nouvelles technologies de l'information et de la communication (NTIC) transforment les modèles de publication et d'édition de la littérature savante autant d'un point de vue technologique que par les modes de diffusion. À part ces considérations technologiques, le spectre des modèles économiques des systèmes de diffusion va de la gratuité de certaines revues et autres publications à l'emprise de quelques groupes d'éditeurs commerciaux qui pratiquent des grilles tarifaires extravagantes.

Quel que soit l'intérêt des serveurs de prépublications, il faut éviter les amalgames. L'objectif premier de ces serveurs, c'est la communication des résultats de recherche des correspondants, sans filtrage à l'entrée, par la diffusion des textes dans l'état où ils sont déposés sur ces serveurs. Ce qui distingue la communication de la publication, c'est que cette dernière existe en raison du processus d'ensemble de sélection, de traitement, de mise en forme, de diffusion, d'institutionnalisation des forums et d'archivage des textes soumis aux revues. Comme le souligne Arms, l'éditeur de D-Lib:

Today, digital libraries and electronic publishing provide authors with many other options, ranging from departmental reports, to eprint archives and electronic journals. While it is clear that these new methods of scholarly communication are widely used, thera bas been little systematic study of how specific disciplines are making use of the alternatives. (Arms 1999)

Par nos activités d'éditeurs, nous travaillons quotidiennement à la production et à la diffusion de revues savantes des disciplines des sciences humaines et sociales publiant traditionnellement sur support papier et qui se trouvent à plusieurs égards au carrefour des différentes possibilités qu'offrent les NTIC ${ }^{3}$. Nous proposons, plutôt que la simple translation de la revue vers l'électronique, la création d'un nouveau modèle pour la revue savante au sein d'un environnement organisationnel sans but lucratif. Ce modèle est basé sur des structures existantes qui occupent, comme nous le démontrons dans cet article, un espace qu'on ne peut pas sousestimer et qui est là, à côté des éditeurs commerciaux et des serveurs de prépublications. Notre démarche vise à proposer un modèle réaliste permettant aux revues, d'une part, d'effectuer la transition vers l'électronique selon un modèle qui répond aux besoins du milieu et de ce type de document et, d'autre part, de contribuer à la mise en œuvre d'un système de diffusion qui assure la viabilité économique des revues, c'est-à-dire qui assure les frais de préparation et de diffusion des textes.

Avant de définir les stratégies d'action en matière de réseaux, il convient de bien cerner les positions relatives des acteurs de l'édition de revues savantes. Pour ce faire, nous retiendrons les principaux titres dans huit disciplines des sciences naturelles et des sciences sociales. De là, nous pourrons identifier les éditeurs, saisir leurs pratiques commerciales et jauger leur poids relatif dans les canaux de communication scientifique. Les données sur le facteur d'impact des revues serviront d'outils pour établir notre échantillon de revues et rendre compte de l'importance relative des revues dans la communauté des chercheurs. 


\section{Caractérisation sommaire de la place des revues}

Afin de rendre compte de certaines caractéristiques de la place des revues dans les disciplines, nous avons retenu la typologie de Becher (1987) qui distingue quatre grands types de champs disciplinaires que sont le hard pure, le hard applied, le soft pure et le soft applied, et associe chaque type à deux disciplines. Dans le premier cas, nous retenons la chimie et la physique, dans le deuxième, le génie électrique et la science des matériaux, dans le troisième, la science politique et la sociologie, et, dans le quatrième, les sciences de l'éducation et le travail social. Pour chaque champ disciplinaire, les 25 revues ayant eu en 1997 le facteur d'impact le plus élevé, selon le $S C I$ Journal Citation Reports et le SSCI Journal Citation Reports, ont été sélectionnées. Le facteur d'impact doit être utilisé avec prudence. Dans le cadre de cette étude, cette mesure a permis de sélectionner les revues qui auraient une position dominante dans les disciplines et d'établir un échantillon qualifié pour chacune.

Les facteurs d'impact des revues des sciences sociales sont beaucoup moins élevés que ceux des secteurs scientifiques et présentent également une moindre disparité élevée. Les facteurs d'impact sont des révélateurs de pratiques et de conditions différenciées de production et de diffusion de la recherche dans les disciplines et rendent compte d'un certain nombre de variables. Pensons au nombre de chercheurs actifs, au nombre d'auteurs, au nombre de périodiques disponibles, publiés et cités, au nombre de références par article publié, à la longueur moyenne d'un article publié, etc. Cela dit, grossièrement, un certain nombre de similitudes semblent se dégager selon qu'on fasse référence aux sciences naturelles ou aux sciences sociales 4 . Par ailleurs, il existe généralement une plus grande concentration des citations dans un petit nombre de revues, conformément à la loi de Bradford.

La demi-vie d'une revue témoigne du nombre d'années de publication qu'il faut rétrospectivement compter pour atteindre la moitié des citations que la revue a obtenues durant l'année courante. La différence de demi-vie des articles entre les deux grands groupes disciplinaires étu-

Tableau 1 : Parts du marché et facteurs d'impact selon le type d'éditeur

\begin{tabular}{|c|c|c|c|c|}
\hline \multirow{2}{*}{..} & \multicolumn{2}{|c|}{ SCIENCES SOCIALES } & \multicolumn{2}{|c|}{ SCIENCES NATURELLES } \\
\hline & $\begin{array}{l}\text { Part du } \\
\text { marché }\end{array}$ & $\begin{array}{l}\text { Facteur } \\
\text { d'impact }\end{array}$ & $\begin{array}{l}\text { Part du } \\
\text { marché }\end{array}$ & $\begin{array}{l}\text { Facteur } \\
\text { d'impact }\end{array}$ \\
\hline Presses universitaires & $26 \%$ & 0,84 & $1 \%$ & \\
\hline Sociétés savantes & $28 \%$ & 1,24 & $55 \%$ & 2,96 \\
\hline Éditeurs commerciaux & $46 \%$ & $0 ; 74$ & $44 \%$ & 2,63 \\
\hline
\end{tabular}

diés, sans être très grande, demeure significative. Dans les sciences sociales, cette dernière est de 7,28 années en moyenne, contre 6,38 années en moyenne dans les sciences 5 . II est intéressant de noter par ailleurs que les revues ayant des demivies supérieures à 10 sont presque aussi nombreuses dans les deux champs. En effet, 14 revues scientifiques ont une valeur de demi-vie supérieure à 10, alors que 12 ont une marque semblable dans les sciences sociales. L'utilisation des revues savantes, autant en sciences naturelles et pures qu'en sciences humaines et sociales, n'est donc pas basée que sur le court terme. L'importance de la conservation et de l'archivage ainsi que de la numérisation rétrospective des collections se posent donc dans les mêmes termes pour les deux groupes disciplinaires.

\section{Structuration économique}

\section{Éditeurs des revues dominantes}

Dans les secteurs scientifiques, représentés par la chimie, la physique, le génie électrique et la science des matériaux, les presses universitaires sont pratiquement absentes: une seule revue du groupe, l'Israel Journal of Chemistry, est éditée par une presse universitaire, celle du Wizemann Institute. Les autres sont partagées entre les sociétés savantes (55\%) et les éditeurs commerciaux (44\%), et ces deux groupes présentent des facteurs d'impact moyens semblables (respectivement $2,96$ et 2,63$)^{6}$.

La situation est quelque peu diffé- rente dans les sciences sociales, représentées par la sociologie, la science politique, les sciences de l'éducation et le travail social. Les revues dominantes de ces disciplines sont beaucoup plus souvent éditées par des presses universitaires, portant la part de ce type d'éditeur à $26 \%$ au sein du regroupement disciplinaire. Les sociétés savantes sont éditrices de $28 \%$ des revues, contre $46 \%$ publiées par des éditeurs commerciaux. Les facteurs d'impact moyens pour les presses universitaires sont de 0,84 , pour les sociétés savantes de 1,24 et de 0,74 pour les éditeurs commerciaux. (Tableau 1)

Les éditeurs commerciaux publient donc un peu moins de la moitié des revues dominantes des champs que nous avons étudiés, tant dans les sciences que dans les sciences sociales. Les presses univer-

4. Pour toutes les disciplines rattachées aux sciences (physique, chimie, science des matériaux et génie électrique), les facteurs d'impact médians étaient toujours supérieurs à 1,46 , ce minimum étant occupé par le génie électrique et le maximum étant représenté par la chimie avec 2,68 . La médiane pour toutes les disciplines se situait à 1,71 , tandis que la moyenne se situe à 2,80 . La situation est très différente en sciences sociales (sociologie, science politique, travail social et éducation), où le facteur d'impact médian le plus fort se situe à 1,04 (sociologie), et le minimum s'affiche à 0,43 (travail social), la médiane globale étant de 0,77 . La moyenne globale se situe pour sa part à 0,90 . L'écart type est de 0,54 , ce qui est significativement plus faible que pour le groupe des disciplines scientifiques $(2,25)$.

5. Rappelons cependant que les demi-yies supérieures à 10 ont été ramenées à 10 pour le calcul de la moyenne, puisqu'on n'en connaissait pas la valeur réelle, le fournal Citation Reports de ISI ne fournissant que la notation $«>10 »$.

6. Ces proportions sont comparables à celles qu'Abate (1997) rapporte : $40 \%$ des revues scientifiques étant produites par des éditeurs commerciaux tandis que les presses universitaires ainsi que les agences gouvernementales publient ensemble $60 \%$ de ce genre de publication. 
sitaires, lorsqu'il y a lieu, et les sociétés savantes se partagent le reste de l'édition savante.

\section{Coûts des abonnements}

D'entrée de jeu, on remarque que le prix moyen 7 des revues en sciences est près de cinq fois plus élevé qu'en sciences sociales, avec des valeurs respectives de $1164,95 \$$ et de $224,76 \$ 8$. Cette différence s'atténue quand on observe les médianes, qui se situent plutôt à $735,00 \$$ et $169,66 \$$ respectivement, portant le rapport à $4: 1$ environ.

Les bibliothécaires ne seront pas surpris de constater que les prix moyens des revues dominantes, lorsqu'ils sont considérés sous l'angle du type d'éditeur, sont particulièrement disparates: si le coût moyen des revues publiées par les sociétés savantes est de $737,47 \$$, celui des revues éditées commercialement grimpe à 1794,29 \$, soit plus du double. Plus encore, ce rapport passe au triple environ quand on compare les médianes, qui sont respectivement de 430,00\$ et de $1351,96 \$$. Notons que la plus chère des publications de notre échantillon éditées par une société savante coûte près de 5000 \$ par an (Journal of Physics $A-M a-$ thematical and General de l'American Institute of Physics), tandis qu'il faut compter un peu plus de $7000 \$$ pour la plus chère des revues commerciales (Physics Letters $B$, publiée chez North-Holland/Elsevier).

Du côté des sciences sociales, le tableau est semblable, même s'il faut tenir compte d'une dynamique à trois groupes - les presses universitaires étant cette fois beaucoup mieux représentées. II reste que ces dernières se retrouvent sensiblement dans les mêmes tarifs d'abonnements que les sociétés savantes, présentant respectivement des moyennes de $118,95 \$$ (presses universitaires) et de $108,60 \$$ (sociétés savantes), et des médianes de $100,00 \$$ et de $98,50 \$$. Ici encore, les revues publiées par des éditeurs commerciaux ont des tarifs plus élevés selon un facteur d'environ 3,3 (moyenne de $337.03 \$$ ) ou 2,6 (médiane de 257,50\$) selon que l'on considère la moyenne ou la médiane des prix. La situation est donc assez semblable à celle des secteurs scientifiques, mais dans un ordre de prix différent. Ce sont, là encore, les éditeurs commerciaux qui détiennent la palme de la revue la plus chère $(1072 \$$ pour la revue
Child Abuse \& Neglect, également un produit Elsevier), suivis des sociétés savantes (308\$ pour Journal of Social Work Education du Council on Social Work Education) et des presses universitaires (265\$ pour Health Education Research de Oxford University Press).

On aurait pu tenir compte du poids relatif des revues: gabarit, complexité et périodicité. En effet, certaines sont publiées une fois l'an, d'autres douze fois ou plus. Certaines comptent quelques centaines de pages annuellement, d'autres sont imprimées en petits caractères sur plúsieurs milliers de feuillets de papier bible. Mais il reste qu'en comparant des revues dans un nombre restreint de disciplines et avec un statut similaire, ces différences s'annulent à l'intérieur des grands champs; elles reprennent de leur pertinence lorsque vient le temps d'apprécier avec précision la justification de l'écart des prix entre les grands champs et ne comptent vraisemblablement que pour une partie de l'explication. De plus, certaines études similaires à la nôtre, mais plutôt préoccupées par l'approfondissement d'un champ particulier de la science, ont livré des résultats semblables. Par exemple, le Scripps Institution of Oceanography Library a établi, pour 1996, le coût moyen par page pour chacun des éditeurs dans le champ de l'océanographie et concluait que: «Society and university publishers tend to have a lower cost per page than commercial publishers but there are exceptions. " (Brueggeman 1996)

\section{Fréquence d'utilisation et impact}

On prétend souvent que les revues publiées par les grands éditeurs commerciaux sont choisies par les bibliothécaires et les universitaires en raison de leur grande consultation et de leur importance dans le domaine. Cette affirmation n'est pas sans susciter quelque scepticisme. Pour sa part, Wilder (1998) soutient que les publications commerciales représentent $75 \%$ des titres de la bibliothèque de chimie de l'University of Illinois (UrbanaChampaign), mais seulement $42 \%$ de l'utilisation (mesurée par le nombre de fois qu'un titre était mis en circulation ou replacé sur les tablettes), tandis qu'avec seulement $21 \%$ des titres, les associations américaines sans but lucratif obtenaient $51 \%$ de l'utilisation. La mesure des citations des revues commerciales de chimie auxquelles la Louisiana State University est abonnée donne des résultats semblables. Pour $78 \%$ des titres, les périodiques d'éditeurs commerciaux recueillent à peine $50 \%$ de l'ensemble des citations attribuées à des périodiques auxquelles cette bibliothèque est abonnée.

La conclusion de Wilder est plutôt lourde, lorsqu'il suggère: «By placing value at the heart of their $S T$ collection development philosophies, academic libraries can free themselves from the highcost, low value commercial titles that currently consume such a large proportion of ST journal budgets. " (Wilder 1998). Cette conclusion, venant par ailleurs du vicedoyen d'une bibliothèque universitaire, est peut-être hasardeuse si l'on tient compte de la base fragile sur laquelle elle est posée. On sait en effet que la discipline de la chimie est caractérisée par une asymptote particulièrement abrupte, illustrant l'importante concentration des citations dans un petit nombre de publications. La simple présence d'un éditeur commercial à la première position de la liste aurait modifié considérablement ses calculs, si bien que ses conclusions risquent d'être moins pertinentes dans d'autres domaines.

Dans la continuité de la démonstration de Wilder et afin d'établir une mesure de la position relative des revues au sein de la communauté des chercheurs selon le type d'éditeur, nous avons attribué à chacun des titres un pointage correspondant à l'inverse de son classement dans son champ de spécialisation. Avec un total de 25 revues par champ, les revues qui se retrouvent au premier rang des facteurs d'impact de leur champ se voient attribuer 25 points contre 1 seul point pour la vingtcinquième revue. Voulant tester l'idée que les revues dotées des plus forts indices sont concentrées chez les grands éditeurs commerciaux, nous avons procédé à l'établissement d'un pointage moyen des revues pour les types d'éditeurs. (Tableau 2)

7. Le coût sur lequel nous basons nos calculs est celui d'un abonnement institutionnel d'une durée d'un an poúr un établissement américain, en devises américaines. Le coût de l'abonnement à la version papier sert de mesure de référence lorsque l'éditeur permet de choisir entre un abonnement à la version papier, à la version électronique ou aux deux.

8. Ces données sont comparables aux résultats publiés par Library fournal en avril 1999. 
Tableau 2: Pointage moyen des revues en fonction de leur impact selon le type d'éditeur

\begin{tabular}{|c|c|c|}
\hline & Pointage moyen & Nombre de revues \\
\hline \multicolumn{3}{|l|}{ Sciences sociales } \\
\hline Presses universitaires & 13,00 & 25 \\
\hline Sociétés savantes & 15,41 & 27 \\
\hline Éditeurs commerciaux & 11,80 & 45 \\
\hline \multicolumn{3}{|l|}{ Sciences } \\
\hline Presses universitaires & 12,5 & 2 \\
\hline Sociétés savantes & 13,23 & 53 \\
\hline Éditeurs commerciaux & 12,82 & 44 \\
\hline
\end{tabular}

Tableau 3: Coûts pour $1 \%$ des citations reçues par les 200 revues de l'échantillon

\begin{tabular}{lcc} 
& & \\
\cline { 2 - 3 } & Sciences sociales & Sciences naturelles \\
\cline { 2 - 3 } Presses universitaires & $36,26 \$$ & $*$ \\
\hline Sociétés savantes & $27,02 \$$ & $256,58 \$$ \\
\hline Éditeurs commerciaux & $100,88 \$$ & $666,32 \$$ \\
\hline Moyenne & $37,85 \$$ & $436,78 \$$ \\
\hline
\end{tabular}

* Cette cellule reste vide puisque l'échantillon (deux revues) ne nous permet pas d'obtenir une valeur fiable.

Tableau 4: Pourcentages des citations en regard de la proportion du budget d'acquisition des 200 revues de l'échantillon

\begin{tabular}{lcccc}
\hline & \multicolumn{2}{c}{ SCIENCES SOCIALES } & \multicolumn{2}{c}{$\begin{array}{c}\text { SCIENCES } \\
\text { NATURELLES }\end{array}$} \\
\cline { 2 - 5 } & $\begin{array}{c}\% \text { du } \\
\text { budget }\end{array}$ & Citations & $\begin{array}{c}\% \text { du } \\
\text { budget }\end{array}$ & Citations \\
\cline { 2 - 5 } & $13 \%$ & $24 \%$ & & \\
\hline Presses universitaires & $13 \%$ & $36 \%$ & $36 \%$ & $56 \%$ \\
\hline Sociétés savantes & $74 \%$ & $40 \%$ & $63 \%$ & $42 \%$ \\
\hline Éditeurs commerciaux & $19329 \$$ & & $103680 \$$ & \\
\hline Budget d'acquisition & & & & \\
\hline
\end{tabular}

On est frappé par le fait que les différents types d'éditeurs se partagent plutôt également le classement et ont une propension à se disperser à peu près également autour de la moyenne présentant tous des écarts types avoisinant 7,5 tant dans les sciences sociales que dans les sciences naturelles. En d'autres termes, les grands éditeurs commerciaux ne semblent pas présenter un avantage particulier pour ce qui est de l'impact relatif de leurs revues dans la communication scientifique.

\section{Mais le prix de l'impact?}

Dans la mesure où le coût des abonnements est beaucoup plus élevé chez les commerciaux et que leur indice d'impact ne se différencie pas de celui des autres types d'éditeurs, il nous a semblé utile de construire un indicateur permettant de mesurer le coût financier d'acquisition de périodiques d'impacts équivalents. Le pointage a été attribué en transposant la valeur du facteur d'impact de chaque revue en pourcentage de la somme des facteurs d'impact. Par la suite, le coût d'abonnement a été divisé par le pointage obtenu pour chacune des revues et des moyennes ont pu être calculées.

Ainsi, au sein de nos huit groupes disciplinaires sélectionnés et pour les 25 premières revues de chaque champ, il coûte en moyenne $37,85 \$$ pour obtenir $1 \%$ de toutes les citations (reçues par les 100 revues sélectionnées) contre 436,78 en moyenne pour la même portion des citations en sciences naturelles. Cette différence de prix est déjà connue. Mais le recoupement qui est fait avec les éditeurs permet d'illustrer, par une autre image (toute financière soit-elle), la différence entre les coûts des publications en fonction du type d'éditeur. En somme, les revues publiées par des éditeurs commerciaux en sciences naturelles coûtent $666,32 \$$ pour $1 \%$ des citations obtenues contre nettement moins de la moitié $(256,58 \$)$ pour les sociétés savantes. La situation en sciences sociales est semblable, comme le démontre le tableau 3.

Cela signifie que, dans une situation où une institution serait abonnée à toutes les revues étudiées, les trois quarts du budget d'acquisition en sciences sociales et les deux tiers en sciences seraient canalisés vers des éditeurs commerciaux pour des publications ne représentant respectivement que $40 \%$ et $42 \%$ des citations dans les divers domaines. Le coût des revues rattachées à des éditeurs sans but lucratif est de toute évidence beaucoup plus modeste, en fonction de leur rayonnement. (Tableau 4)

Tout en n'étant qu'indicatives, ces données font ressortir que, peu importe la méthode de calcul, les revues publiées par des éditeurs commerciaux, à qualité égale, coûtent beaucoup plus cher que les revues éditées par des sociétés savantes ou des presses universitaires, tant en sciences naturelles qu'en sciences humai- 
nes et sociales. II en ressort également que les publications des éditeurs commerciaux ne se positionnent pas plus avantageusement que leurs concurrentes sans but lucratif, dans les huit champs que nous avons explorés simultanément, quoique certains domaines soient habités par un type de revue plutôt que par un autre.

Les distinctions entre les pratiques des éditeurs aident à avoir une vision plus circonstanciée de la place de ceux-ci en fonction des augmentations de coûts et de la spirale inflationniste qui mine l'accès à l'information scientifique, de même qu'elles soulignent le caractère hasardeux voire dangereux de généralisations abusives. Elles permettent également des rapprochements extrêmement convaincants dans les pratiques des éditeurs sans but lucratif (sociétés savantes comme presses universitaires). Il est utile maintenant de se pencher plus attentivement sur le rôle de l'éditeur et sur les enjeux auxquels il fait face dans l'environnement de la publication électronique.

\section{La valeur ajoutée par l'éditeur et son champ d'intervention}

L'éditeur apporte une valeur ajoutée pertinente et significative, tant pour le processus d'édition que pour son intervention dans le contexte de l'électronique.

Avec les nouvelles technologies de l'information, de très nombreuses initiatives ont vu le jour afin d'exploiter le potentiel énorme que recèle la communication électronique, sans pour autant recourir aux procédures de publication et d'édition. C'est ainsi que l'accès libre donné aux textes par les auteurs est devenu monnaie courante. Ce modèle de publication ne répond toutefois pas à la recherche de normalisation et de reconnaissance qui est présente dans le processus d'édition.

La méconnaissance du rôle de l'éditeur contribue souvent à en sous-estimer l'importance. Pour qui ce rôle se résumerait à celui d'intermédiaire entre l'auteur et l'imprimeur, les nouvelles techniques de publication peuvent signifier la mise en cause du statut de l'éditeur. En fait, l'édition universitaire, électronique notamment, fait référence au processus d'institutionnalisation des forums d'échange scientifique. Cela suppose une série d'opéra- tions sur plusieurs plans.

Considérons quatre dimensions du rôle conventionnel de l'éditeur universitaire:

La première concerne l'évaluation et la sélection des manuscrits. Dans le cas des revues, ce rôle est, de fait, délégué au comité de rédaction ou de direction de la revue, mais l'éditeur reste garant de la qualité et veille à ce que le comité de rédaction s'acquitte bien de ses responsabilités.

- La deuxième porte sur le traitement et la mise en forme du texte. L'intervention concerne aussi bien la construction du texte que sa correction dans l'expression écrite. S'ajoute à cela la présentation matérielle de la publication: la rigueur de sa présentation, l'uniformité dans l'application de la grille typographique, la qualité et la pertinence du choix des matériaux et le caractère irréprochable de la mise en forme.

Ea troisième touche la diffusion, la promotion et la vente. L'éditeur s'occupe de la mise en marché, de la promotion et de la distribution afin de faire en sorte que les auteurs joignent le plus grand nombre de lecteurs possibles.

La quatrième dimension prend du relief en fonction des enjeux actuels concernant l'archivage. L'éditeur conventionnel n'assume pas véritablement un rôle à ce titre. II lui incombe cependant de garder un inventaire complet de ses publications afin de répondre à la demande de numéros anciens. L'archivage est assuré par les bibliothèques nationales, par la diffusion géographique des exemplaires dans de multiples lieux et par le réseau des bibliothèques de recherche. En ce sens, l'archivage pour l'éditeur conventionnel consiste essentiellement à la constitution et à la préservation de son fonds d'archives historiques d'entreprise.

Toutes ces dimensions sont entièrement présentes dans le contexte de l'appropriation par les éditeurs des technologies de l'information et de développement de la publication électronique. Ces missions conventionnelles s'enrichissent d'ailleurs de façon très nette.

\section{L'électronique enrichit le travail d'édition}

Le traitement, la mise en forme et la diffusion de la documentation scientifique imposent de procéder à des choix technologiques, notamment pour les formats, ce qui relève davantage de la conception de l'édition en fonction de la qualité des textes à publier que d'éléments d'abord techniques. L'éditeur doit aussi avoir une implication dans l'exploitation des NTIC, ce qui peut se traduire par l'indexation de la revue, par l'ajout de liens hypertextes, par l'aménagement d'un espace de communication (commentaires, listes de discussion), par l'utilisation du multimédia (image, son, vidéo).

Par ailleurs, l'éditeur doit veiller, en plus des modes traditionnels et de leur adaptation en fonction de la diffusion sur Internet, à doter son site de services qui vont faciliter la diffusion et optimiser l'exploitation des textes mis en ligne. C'est ainsi que la mise en place d'un outil de recherche performant s'avère indispensable et qu'il est fort à propos d'envisager d'autres services, tels que l'utilisation de listes de diffusion ou la diffusion sélective d'information. De plus, partant du fait que l'éditeur ne vend plus un produit tangible, mais offre un service ou un accès à de la documentation, il doit assurer une stabilité et une qualité d'accès à cette documentation.

Enfin, l'éditeur ne peut échapper à sa responsabilité concernant l'archivage des documents mis en ligne. II doit s'assurer de la pérennité de l'information éditée et diffusée, et prendre des dispositions garantissant l'accès, sur une longue période, au service offert. Cela dépasse largement sa pratique traditionnelle de gestion d'archives historiques d'entreprise et le plonge de plain-pied dans une problématique d'archivage et de conservation.

II est utile de reprendre certaines de ces considérations en partant d'un simple constat. Lorsque l'on consulte un document électronique, on est de prime abord frappé par l'absence de distinctions physiques entre les types de documents, alors que ces distinctions sautent aux yeux avec les documents imprimés. Ainsi cette absence, dans le cas de documents électroniques, ne permet pas de distinguer a priori une monographie, d'un périodique ou d'une encyclopédie. L'écran reste, en grande partie, la seule interface entre le lecteur et l'information, que ce soit pour un journal, une revue savante ou une base de données. Cela provoque d'ailleurs très souvent l'illusion de la facilité de traitement et de production des documents électroniques.

Pour l'électronique, les caractéristiques de l'édition se révèlent dès qu'on dépasse la simple consultation à l'écran et 
que l'on cherche, par exemple, à exploiter les possibilités de recherche ou à réutiliser les données. Comme pour l'édition imprimée conventionnelle, l'éditeur doit faire des choix technologiques et artistiques correspondant aux caractéristiques du document qu'il a à publier et à diffuser. La revue savante, par exemple, a pour caractéristiques de se présenter comme une information textuelle, souvent en plusieurs langues dans un même document, avec des tableaux et des formules mathématiques complexes, avec une iconographie (graphiques ou photographies) noir et blanc et couleurs, avec l'utilisation de l'hypertexte et de l'hypermédia. Cela nécessite, d'abord, l'emploi d'un format d'encodage puissant. Ensuite, l'importance de ce type d'information pour la communauté des chercheurs et pour la société en général impose des choix technologiques permettant l'archivage et la conservation pour les futures générations. Le format d'encodage retenu doit, enfin, permettre des possibilités de recherche qui diminuent le plus possible le bruit et le silence.

En ce sens, une chaîne de traitement pour la publication et la diffusion électroniques de la littérature universitaire doit s'appuyer sur un format performant, très stable et qui permet de produire automatiquement des formats et des produits dérivés. II en ressort que, sans que les moyens à mettre en œuvre pour assurer une publication et une diffusion électroniques de qualité soient inaccessibles et d'une complexité effarante, l'édition électronique demande une expertise et un savoir-faire professionnels, tout comme c'est le cas pour l'édition imprimée.

Peu importent les habiletés des auteurs à utiliser les outils informatiques pour la rédaction de leurs textes, l'éditeur se voit confirmer dans son rôle. Supposons un instant que tous les auteurs soient en mesure de préparer des documents électroniques de qualité. Chacun de ces documents sera créé de façon différente dans la présentation et les formats des données textuelles (de toutes langues), iconographiques ou sonores. L'éditeur a le mandat de normaliser et de préparer ces données pour leur diffusion et leur conservation. L'édition électronique renvoie à bien plus que la simple page HTML; les développements se produisent à un rythme accéléré, comme dans tous les domaines, et les auteurs ont mieux à faire que de suivre et appliquer les particularités des
CSS, du XML, des métadonnées ou d'Unicode.

\section{Bien cerner les nouveaux enjeux pour l'éditeur}

Tout en ayant un fond de continuité et de nombreuses analogies avec le métier et les habiletés de l'édition conventionnelle, les nouvelles technologies reliées à la publication et à la diffusion électroniques amènent les éditeurs à reconsidérer de fond en comble leurs activités. Quatre enjeux ressortent particulièrement.

Le premier enjeu consiste à mettre en place une chaîne de traitement électronique permettant la production de produits dérivés. Le deuxième enjeu porte sur les modalités de diffusion de l'électronique. La conservation, l'archivage et l'indexation - fonctions jusqu'à maintenant assurées par les bibliothécaires - deviennent la responsabilité de l'éditeur. Ce déplacement de la frontière entre les rôles encourage des alliances qui permettent la mise en commun d'expertises. Les moyens de diffusion et de mise en marché, par la nature différente de la production de l'éditeur, sont à revoir et à considérer, ce qui constitue en soi un troisième enjeu important. Des modifications majeures aux activités et aux pratiques actuelles sont à prévoir afin d'assurer une diffusion active et d'exploiter des marchés jusqu'ici très difficiles à atteindre. Le quatrième enjeu est sans doute très stimulant pour l'éditeur dans la mesure où il participe à la création de nouveaux modèles de transmission de la connaissance qui mettent à profit l'interaction entre les lecteurs et les auteurs ainsi que l'utilisation du multimédia. Ce dernier enjeu mérite quelques considérations supplémentaires.

L'édition électronique, telle qu'elle est pratiquée jusqu'à maintenant, est le plus souvent une simple transposition du document conçu et présenté pour sa version imprimée. Force est de constater, partant de notre expérience et de la consultation de ce qui est disponible sur le Web, qu'il y a une grande sous-utilisation des nombreuses possibilités offertes par la nature électronique du document. Pensons à l'indexation en texte intégral, à l'inclusion de données actives, à l'établissement de liens hypertextes, à l'ouverture de forums pour les commentaires autour des articles, à l'utilisation des ressources du multi- média (image, son, vidéo, simulation 3D), etc. (Le Crosnier 1997). II y a là un paradoxe assez frappant, car, outre le mode de diffusion par Internet, ce sont ces possibilités particulièrement intéressantes que l'on met généralement de l'avant pour souligner l'attrait et la grande puissance de l'édition électronique par comparaison à l'édition imprimée, mais dans les faits, ces possibilités, qui constituent une véritable valeur ajoutée (Raney 1998), restent la plupart du temps du domaine de la virtualité. Or, l'éditeur a un rôle important à jouer pour créer de nouveaux modèles d'organisation et de transmission de l'information et faire en sorte que, au moment même de rédiger son document, l'auteur ait en tête sa diffusion électronique.

Ainsi, le défi posé à l'éditeur ne relève pas tant de la réalisation technique d'un système de publication électronique, mais découle surtout de la mise en place d'un système d'exploitation des possibilités offertes par la version électronique des textes, de production et d'édition de qualité, de conservation, d'archivage et d'indexation et, finalement, de diffusion systématique et de services.

\section{La problématique des réseaux}

Une idée simple condense certains enjeux importants qui doivent être traités avec attention. Cette idée, c'est celle de la problématique des réseaux. Manuel Castells $(1998,525)$ observe que «les fonctions et les processus dominants de l'ère de l'information s'organisent de plus en plus en réseaux. Les réseaux constituent la nouvelle morphologie sociale de nos sociétés, et la diffusion de la logique de la mise en réseau détermine largement les processus de production, d'expérience, de pouvoir et de culture [...] [Ce] qui est nouveau aujourd'hui, c'est que le nouveau paradigme des technologies de l'information fournit les bases matérielles de son extension à la structure sociale tout entière. "

Parmi les composantes de ce paradigme, notons l'omniprésence des effets des nouvelles technologies, la logique en réseau de tout groupe de relations qui utilise ces nouvelles technologies de l'information et la convergence croissante des technologies particulières au sein d'un système hautement intégré (Castells 1998, 87 et suivantes). De l'observation 
des tendances lourdes de l'évolution des sociétés actuelles sous l'influence des nouvelles technologies de l'information, on peut dégager des directions et même des stratégies d'action.

\section{Stratégies d'action en phase avec les auteurs}

II ressort assez clairement qu'une direction de revue isolée peut difficilement, pour employer un euphémisme, se donner les moyens pour assurer la publication et la diffusion électroniques de trois ou quatre numéros par année, à un coût et dans des conditions de réalisation qui soient raisonnables. À première vue, un volume d'opération s'impose pour permettre de réunir les ressources et pour assurer une visibilité et une démarche de vente qui soient efficaces. La question peut être posée de façon banale, sous l'angle du simple seuil de rentabilité. II vaut sans doute mieux considérer cette variable « technique » en référence au cadre organisationnel qui pourrait permettre la publication de qualité et une présence forte dans les circuits mondiaux.

Afin d'offrir une structure d'accueil propice à tout regroupement des efforts, il importe qu'elle se modèle à partir des principes d'une société sans but lucratif. C'est sûrement la forme la plus adaptée à la mission universitaire. On peut même imaginer qu'avec une organisation efficace, c'est le tremplin qui est prédestiné à faire contrepoids et même concurrence à l'édition commerciale de documents universitaires. Dans tous les cas, c'est l'environnement qui peut le mieux offrir un service de qualité dans des normes de coûts acceptables.

L'enjeu majeur, incontournable, implacable, c'est la capacité de mettre en place une démarche qui soit enracinée dans le milieu et qui rende compte de ses caractéristiques. Pour que l'action soit pertinente et efficace, elle doit reposer sur une vue circonstanciée qui dépasse le niveau macroscopique et qui tienne compte de la double structuration des canaux de communication. Les grilles tarifaires pour les abonnements sont certes une variable très importante. Toutefois, en tenant compte également du deuxième élément de la structuration du milieu qui se rapporte aux usages de la communication scientifique selon les secteurs, nous pouvons mettre en œuvre une stratégie d'ac- tion en phase avec les acteurs déjà en place.

Comme le démontre notre étude, les sociétés savantes et les presses universitaires occupent une place importante dans la publication des revues en sciences pures et en sciences sociales tant par le nombre de titres publiés que par leur importance et leur qualité. L'organisation de l'édition montre que des éditeurs sans but lucratif ont des pratiques qui ne contribuent pas à la crise financière de l'édition savante et qu'ils jouent un rôle de premier plan parmi les revues dominantes. Le travail d'édition, loin de s'effacer, se transforme en s'enrichissant et permet de penser de nouvelles relations avec des intervenants nouveaux ou se présentant dans des termes renouvelés, notamment avec les bibliothèques.

Plusieurs projets d'édition électronique proviennent du milieu des bibliothèques $^{9}$. Que ce soit par le biais de la numérisation des collections spéciales ou de projets de diffusion électronique des thèses, les possibilités des NTIC amènent de plus en plus les bibliothécaires à s'intéresser aux activités d'édition. Toutefois, le rôle que les bibliothèques doivent jouer pour la mise en œuvre des bibliothèques virtuelles tient également à leur mission conventionnelle et doit faire en sorte que la valeur ajoutée de la chaîne documentaire soit transposée aux nouvelles formes de diffusion de l'information.

\section{La bibliothèque virtuelle de Babel}

Quelles sont les caractéristiques de la bibliothèque traditionnelle que nous voulons retrouver dans une bibliothèque numérique? Entre l'organisation systématique des ressources documentaires des bibliothèques traditionnelles, l'espace fabuleux ouvert à tous, pour tous les goûts, sous toutes les formes que permet Internet, Harter (1997) propose d'inscrire dans cette Babel virtuelle des espaces où nous retrouverons certaines caractéristiques des bibliothèques traditionnelles. Ces «zones de contrôle» (control zones) donneraient accès à des documents sélectionnés par des spécialistes pour leur qualité et auxquels nous aurons accès par des outils de recherche et des représentations (surrogates), métadonnées ou autres, développés par une chaîne documentaire ap- portant une valeur ajoutée.

Plutôt, ce que nous retrouvons le plus souvent a les allures des galeries hexagonales de la bibliothèque de Babel de Borges. Connaissez-vous ou avez-vous sous la main la liste des serveurs, code d'usagers et mots de passe des éditeurs et autres fournisseurs de services auxquels votre bibliothèque universitaire est abonnée? En plus de se construire littéralement une base de données comportant toutes les informations relatives aux accès, les usagers des bibliothèques ont souvent à butiner d'un serveur à un autre pour arriver à trouver l'information qu'ils cherchent. Les besoins sont pourtant simples à définir : un guichet unique, un portail permettant l'accès à toutes les bases de données textuelles auxquelles une bibliothèque est abonnée. À première vue, on pourrait penser, selon le modèle traditionnel de la bibliothèque, que la solution est de disposer de tous les documents électroniques sur un serveur local. Malgré les problèmes de droits, d'espace disque faramineux et de gestion qu'une telle entreprise représente, le projet TORPEDO du Laboratoire de recherche naval aux États-Unis offre de cette façon 500 revues électroniques (Stackpole et King 1999). La presque totalité des bibliothèques de recherche n'a pas les moyens d'offrir ce genre de service.

La solution visant à offrir la recherche et l'accès au plein texte ne réside pas dans une application Z39.50. II n'est pas illusoire de penser qu'une bibliothèque, ou un consortium de bibliothèques, puisse mettre en place un outil de recherche comme on en trouve des dizaines sur le Web, qui parcoure les bases de données textuelles auxquelles l'institution est abonnée. Les bibliothécaires auraient ainsi un contrôle sur les caractéristiques et les fonctions de l'outil de recherche et seraient plus à même d'exiger des fournisseurs d'information des modèles spécifiques et adéquats de structuration de l'information en vue de sa diffusion et de sa conservation. II s'agit de créer en quelque sorte une «zone de contrôle » fiable et répondant aux besoins des chercheurs et des étudiants, tout comme l'OPAC l'est

9. Notons ici, par exemple, les activités des Electronic Text Centers de l'Université de Virginie, de l'Université du Nouveau-Brunswick, SPARC ou le Scholarly Communications Project de Virginia Tech <URL: http://scholar.lib.vt.edu/ejournals/ > 
pour les ressources documentaires, le plus souvent intra-muros.

Les revues savantes ont quitté le giron du milieu universitaire au début des années 1970 au moment où l'explosion documentaire désorganisait les structures d'édition qui avaient jusqu'alors assuré la diffusion des résultats de la recherche. On connaît la suite.

Le même phénomène est à se reproduire avec la création des portails. Le service Information Quest de Dawson en est un exemple10. Ce type de service permet, après qu'une institution s'y est abonnée, d'effectuer des recherches plein texte ou par champs sur un ensemble de bases de données textuelles pour lesquelles Dawson a négocié une entente avec les propriétaires. Les résultats des requêtes de recherche amènent, soit à accéder au texte de l'article en ligne, s'il est disponible et si votre bibliothèque est abonnée à la revue en question, soit à télécharger ou à recevoir une télécopie moyennant paiement. D'une part, la bibliothèque se retrouve donc à payer pour un abonnement à un outil de recherche «intégrateur" en plus des multiples abonnements aux différentes bases de données d'articles provenant des éditeurs. D'autre part, et c'est peut-être l'enjeu réel à long terme, en adhérant à ce genre de service plutôt que de le développer elles-mêmes, les bibliothèques ne se retrouvent-elles pas, en plus de ne plus avoir de rôle significatif pour le choix, l'archivage et la conservation à laisser à l'entreprise privée le rôle qui leur revenait depuis l'origine de la profession, c'est-à-dire l'analyse, l'organisation, le traitement ainsi que la diffusion de l'information? Dans un tel modèle, les bibliothèques ne se retrouvent-elles pas à n'être que de simples négociatrices de licences de site?

Les bibliothèques ont plutôt à se positionner comme lieu de diffusion et de publication dans une perspective réseau avec les différents acteurs que sont les revues, les éditeurs, dont ceux sans but lucratif (presses universitaires et associations) et le réseau déjà existant des bibliothèques de recherche.

\section{Des lieux de publication en réseau}

Le monde des revues savantes n'est pas contrôlé entièrement par les éditeurs commerciaux, et ce, même en ce qui a trait, comme en témoigne notre étude, aux 25 premières publications qui obtiennent les plus forts facteurs d'impact. Les «lieux d'édition sans but lucratif » sont des acteurs importants pour les revues phares de plusieurs disciplines et probablement prédominants pour les titres de première et de deuxième périphéries. Dans la perspective où les réseaux constituent un mode organisationnel qui traverse la société, une politique de concentration de ressources et de réseaux constitue un intérêt stratégique pour développer une alternative aux oligopoles. II faut donc considérer avec attention la possibilité de développer, d'une part, des pôles de publication et de diffusion électroniques et imprimés de revues et, d'autre part, un réseau de lieux de publication sans but lucratif dont les avantages touchent à la fois la publication et la diffusion.

À un premier niveau, la constitution de lieux de publication doit s'opérer dans le respect de l'autonomie des revues sur les plans éditorial, financier et organisationnel. Le lieu de publication comme première forme de regroupement des revues se distinguerait d'un modèle conventionnel de prestation de services dans une entreprise. Certaines caractéristiques y contribueraient: une structure organisationnelle à but non lucratif, la volonté de ne pas exproprier le contrôle des partenaires, la mise à contribution des partenaires à l'élaboration de certaines solutions concernant la définition des orientations et la recherche de normes de prestations de service qui soient compétitives avec les meilleurs lieux dans le domaine. Ce type réseau pourrait canaliser l'intérêt des revues et proposer aux composantes de contribuer au façonnement de son itinéraire.

À un deuxième niveau, sous une autre forme, le réseau peut prendre la forme d'une collaboration assez étroite d'éditeurs (ou de lieux de publication) qui, au delà de leurs choix éditoriaux et de leur personnalité, peuvent mettre en concordance la conception du travail d'édition sur support électronique, les procédés de publication, les instruments de travail et les modalités de diffusion. De plus, les lieux de publication peuvent établir des normes communes pour la production des métadonnées se rapportant aux articles mis en ligne, ce qui permet de constituer une base de données unique, d'avoir un guichet unique pour la consultation des arti- cles - ce qui n'exclut pas la présence de diverses portes d'accès - et de mettre en place un instrument de recherche performant pouvant balayer un corpus riche, car composé des parutions de nombreuses revues.

Deux conditions sont indispensables pour mettre en place un tel réseau. D'abord, les éditeurs ou lieux de publication doivent individuellement pouvoir compter sur un volume d'activité suffisant pour soutenir la production de publications électroniques et sur les ressources qui sont nécessaires ainsi que sur des compétences pour s'acquitter du travail de publication selon les standards fixés. Ensuite, ils doivent compter sur un code de communication (au sens large) et un cadre de référence commun ou étroitement compatible, cela tant pour la norme de publication que pour les services à offrir aux lecteurs-usagers des revues. Cela n'entraîne pas une uniformité, mais une mise en phase ou une concordance des pratiques dont les paramètres doivent être élaborés et mis en œuvre en collaboration avec les bibliothèques pour assurer la compatibilité avec leurs processus de traitement et de diffusion des revues savantes.

En somme, la mise en commun d'information, la capacité de puiser dans un corpus riche et diversifié, la volonté de se doter de compétences professionnelles, qui se situent à la frontière technologique, la livraison de services plus performants et personnalisés, l'accroissement des moyens de diffusion par un démarchage plus efficace et adapté auprès des institutions invitent à la participation à des réseaux de structures d'édition en relation avec les réseaux des bibliothèques, dont les bénéfices se manifestent dans la production, la consultation, la diffusion et la conservation des revues.

\section{Développer des expertises complémentaires}

Une discussion féconde sur la transformation de la communication scientifique, et sur la place de la publication électronique des revues en particulier, doit pouvoir s'appuyer sur l'identification des ac-

10. <http://www.InformationQuest.com/> 
teurs qui sont à l'œuivre et sur la reconnaissance de leurs positions respectives. Audelà des acteurs, la qualification des rôles dans le contexte de l'appropriation des NTIC permet de cerner la nature des interventions dans le processus de publication et de diffusion des résultats de recherche. Partant des acteurs et des rôles, il est plus aisé de dégager des stratégies dans la constitution de réseaux pour le développement de la publication savante qui émane du milieu universitaire et soit à son service.

Les éditeurs commerciaux de revues ont pris le contrôle de publications dominantes dans les divers secteurs et ont élaboré, à partir d'une position oligopolistique, une stratégie de commercialisation leur permettant à la fois d'imposer leur bassin de revues et de pratiquer des prix extravagants. Cette force commerciale ne doit pas occulter la présence plus que significative des acteurs sans but lucratif; toutefois, elle souligne l'éclatement des modes d'organisation de la publication scientifique chez ces acteurs. Tout en se démarquant des pratiques oligopolistiques, des stratégies de réseautage à différents niveaux permettraient d'allier la force de la cohésion et les personnalités des multiples acteurs.

Les rôles remplis par les différents acteurs ne sont pas incrustés sous une forme organisationnelle immuable. Les lieux de publication, qui ont pris la forme de revues indépendantes (de sociétés savantes ou d'institutions de recherche) orchestrant toutes les opérations, ou encore de maisons d'édition universitaires ou, plus récemment, de services associés aux bibliothèques, ont connu des formes organisationnelles diverses. Dans le contexte actuel, ces formes se renouvellent au gré des innovations et des initiatives. L'édition et la publication évoluent également dans leur mission et dans les pratiques, mais occupent toujours une place capitale dans la communication scientifique. La chaîne qui va de l'édition à la diffusion prend de nouvelles dimensions et redéfinit les points de jonction, mais aussi les pratiques des acteurs, en particulier des bibliothèques.

L'établissement des formes organisationnelles et la façon dont les auteurs définiront leur rôle et s'en acquitteront ne répondent à aucun diktat. Peut-on espérer simplement que l'on puisse profiter des habilités et des expertises de chacun et que la mise en place de réseaux, comme option stratégique, permettra le développement d'expertises complémentaires et non compétitives dans une chaîne constituée de foyers d'excellence composant les diverses mailles de la publication et de la diffusion électroniques.

\section{Sources consultées}

Abate, Tom. 1997. Publishing scientific journals online. BioScience [en ligne] 47 (3). <URL: http:// www.aibs.org/latitude/latpublications.html >

Arms, William Y. 1999. Scholarly communication, digital libraries, and $D$-Lib Magazine. D-Lib [en ligne] 5(4). <URL: http://www.dlib.org/dlib/april99/04editorial.html >

Becher, Tony. 1987. The disciplinary shaping of the profession. In The academic profession: national, disciplinary, and institutional settings, sous la direction de Burton R. Clark. Berkeley: University of California Press, p. 271-303.

Blumenstyk, Goldie et Vincent Kiernan. July 1999. Idea of on-line archives of papers sparks debate on future journals: Proposal by the NIH director infuriates some and inspires others. Chronicle of higher education: A25.

Borgès, Jorge Luis. 1957. Fictions. Paris: Gallimard.

Brueggemant, Peter. 1996. Cost per Page for 1996 subscriptions costing over $\$ 500$ at SIO Library. [en ligne] Site Web de la Scripps Institution of Oceanography Library. <URL: http://scilib.ucsd.edu/sio/guide/prices/perpage.html>

Castells, Manuel. 1998. La société en réseaux. L'ère de linformation. Paris: Fayard.

Hacking, lan. 1996. The disunities of the sciences. In The disunity of science: boundaries, contexts, and power, sous la direction de Peter Galison et David J. Stump. Stanford: Stanford University Press, p. 37-74.

Harnad, Stevan. 1997. The paper house of cards (and why it's taking so long to collapse). Ariadne [en ligne] issue 8. <URL: http://www.ariadne.ac.uk/ issue $8 / \mathrm{harnad} />$

Harter, Stephen P. 1997. Scholarly communication and the digital library: problems and issues. JoDI [en ligne] Volume 1 issue 1 . <URL: http:// jodi.ecs.soton.ac.uk/Articles/v01/101/Harter/>

Institute for Scientific Information. 1997. Science Citation Index. Journal Citation Reports.

- 1997. Social Sciences Citation Index. Journal Citation Reports.

1999. Periodical price survey 1999. Library Journal april 1999.

Le Crosnier, Hervé. 1997. Avons-nous besoin des journaux électroniques? Communication présentée aux Journées SFIC-ENSSIB, Une nouvelle donne pour les journaux scientifiques Villeurbanne, 20 novembre 1997. [en ligne] <URL: http://www.info.unicaen.fr/ herve/pub97/ens sib/enssib.html>

Price, Derek J. de Solla. 1967. Communication in science: the ends-philosophy and forecast. In Communication in science: documentation and automation, sous la direction de Anthony De
Reuck et Julie Knight. Londres: J. \& A. Churchill, p. $199-209$.

Raney, Keith R. 1998. Into a glass darkly. The Journal of Electronic Publishing [en ligne] 4 (2).

<URL: http://www.press.umich.edu/jep/04-02/ raney.html >

Rowland, Fytton. 1997. Print journals: fit for the future? Ariadne [en ligne]. issue 7. <URL: http:// www.ariadne.ac.uk/issue7/fytton/ >

Stackpole, Laurie E. et Richard James King. 1999. Electronic journals as a component of the digital libra$r y$. Issues in Science and Technology Librarianship [en ligne]. Spring 1999, number 22. <URL: http://www.library.ucsb.edu/istl/99-spring/article1.html>

Tenopir, Carol et Donald W. King. 1998. Designing electronic journals with 30 years of lessons from print. The Journal of Electronic Publishing [en ligne] 4(2). <URL: http://www.press.umich.edu/ jep/04-02/king.html >

Wilder, Stanley J. (1998). Comparing value and estimated revenue of scitech journals, ARL Newsletter of Research Library Issues and Actions [en ligne] numéro 200. <URL: http://www.arl.org/ newsitt/200/wilder.html >

Wyly Brendan J. (1998). Competition in scholarly publishing? What publisher profits reveal. ARL Newsletter of Research Library Issues and Actions [en ligne] $\mathrm{n}^{\circ} 200$. <URL: http:ll www.arl.org/newsltr/200/wyly.html > 